\title{
Solidification along the interface between demixed liquids in monotectic systems
}

\author{
C. Hüter, ${ }^{1}$ G. Boussinot, ${ }^{2}$ E. A. Brener, ${ }^{2}$ and D. E. Temkin ${ }^{2}$ \\ ${ }^{1}$ Computational Materials Design Department, Max-Planck-Institut für Eisenforschung GmbH, D-40074 Düsseldorf, Germany \\ ${ }^{2}$ Peter Grünberg Institut, Forschungszentrum Jülich, D-52425 Jülich, Germany
}

(Received 18 February 2011; published 12 May 2011)

\begin{abstract}
The steady-state solidification along the liquid-liquid interface in the monotectic system is discussed. A boundary-integral formulation describing the diffusion in the two liquid phases is given and the corresponding equations for the three interfaces (two solid-liquid interfaces and one liquid-liquid interface) are solved. Scaling relations are extracted from the results and supported by analytic arguments in the limit of small deviation from the monotectic temperature. We present also a complementary phase-field simulation, which proves the stability of the process.
\end{abstract}

DOI: 10.1103/PhysRevE.83.050601

PACS number(s): 68.08.-p, 64.70.dg

\section{INTRODUCTION}

Alloys exhibiting a monotectic point have been of increasing interest in recent years. Important applications of such systems comprise bearings, electrical contacts, and switches. In these systems the three-phase equilibrium consists of two liquids and one solid phase. The physical processes involved in the corresponding phase transitions below or above the monotectic temperature are diverse. In addition to diffusion of impurities, one can, for example, have to deal with convection or gravity effects. For a review of the physics in monotectic systems we refer to Ref. [1]. Here we restrict our interest to diffusional processes.

At the monotectic temperature the three-phase equilibrium consists of two liquids $L_{1}$ and $L_{2}$ and one solid phase $S$ of respective concentrations $c_{1}, c_{2}$, and $c_{S}$. Below the monotectic temperature the $S+L_{2}$ mixture is in stable equilibrium. The liquid-liquid $L_{1}+L_{2}$ equilibrium and the solid-liquid $S+L_{1}$ equilibrium are metastable. The phase transition is controlled by the fluxes in $L_{1}$ (whose concentration is $c_{1 S}$ in equilibrium with $S$ and $c_{12}$ in equilibrium with $L_{2}$ ) and in $L_{2}$ (whose concentration is $c_{2 S}$ in equilibrium with $S$ and $c_{21}$ in equilibrium with $L_{1}$ ). In Fig. 1 we present the corresponding phase diagram.

The similarity between this phase diagram and the eutectic phase diagram makes the classical lamellar growth a usual process for the solidification of monotectic systems. A Jackson-Hunt-type theory is then used to describe this transformation [2]. For hypermonotectic concentrations (i.e., larger than the monotectic concentration $c_{1}$ ), the temperature gradient used in directional solidification experiments allows the system to visit the $L_{1}+L_{2}$ two-phase region and a phase separation of the liquid can appear ahead of the solidification front. Good qualitative agreement with experiments [3] for these processes has been provided by phase-field simulations [4]. In the limit of a vanishing temperature gradient, one can imagine that the $L_{1}+L_{2}$ mixture equilibrates far ahead of the solidification front and the solid grows along an equilibrium liquid-liquid interface. This is precisely what we study in this Rapid Communication, i.e., the isothermal steady-state growth of a finger of solid phase $S$ along the metastable $L_{1}-L_{2}$ interface (see Fig. 2). We consider a two-dimensional pattern with the triple junction as a single point. Asymptotically far ahead of the triple junction, the $L_{1}-L_{2}$ interface is aligned with $y$, the direction of the velocity $v$. The triple junction does not have the same position in the $x$ direction as the asymptotic $L_{1}-L_{2}$ interface, the difference being denoted by $a$. Moreover, at the triple junction, there is an angle $\delta$ between the $L_{1}-L_{2}$ interface and the velocity direction. Asymptotically far behind the triple junction, the solid exhibits an Ivantsov parabolic shape [5] with, in general, a different Péclet number on each side of the finger.

To study this pattern, we solve a boundary-integral equation for each interface $\left(S-L_{1}, S-L_{2}\right.$, and $\left.L_{1}-L_{2}\right)$ that is nontrivial owing to the different nature of the interfaces (solid-liquid and liquid-liquid). We use the framework of the one-sided model together with that of the symmetric model. We present the solution depending on the driving force, i.e., the deviation from monotectic temperature, and show that scaling arguments can be given by the analysis of the equations in the limit of small driving force. We also present the result of a phasefield calculation that demonstrates the stability of the process studied by the boundary-integral equations.

\section{BOUNDARY-INTEGRAL FORMULATION}

In the symmetric model designed for the solidification of a pure material, when the diffusion coefficient is equal in the solid and in the liquid, the diffusion field at the point $(x, y)$ is calculated through the integral along the interface (boundary integral) $y^{\prime}\left(x^{\prime}\right)$ of the Green's function $g\left(x, y ; x^{\prime}, y^{\prime}\right)$. In the one-sided model designed for the solidification of a binary alloy, when the diffusion coefficient vanishes on the solid side of the interface, the boundary integral's kernel depends also on the value of the diffusion field at the interface. In the symmetric model, this dependence is absent because of the continuity of the field (the temperature in most cases). In the most general case where the diffusion coefficient is finite and differs from one phase to another, the kernel of the boundary integral also includes a dependence on the normal derivative of the diffusion field on both sides of the interface. In the symmetric and onesided models this dependence is removed by the incorporation of the energy-conservation law at the moving interface.

Here we assume that the diffusion coefficient vanishes in the solid phase $S$. Hence one integrates along the solid-liquid interfaces a kernel written in the spirit of the one-sided model. Moreover, we consider that the diffusion coefficient is $D$ in 


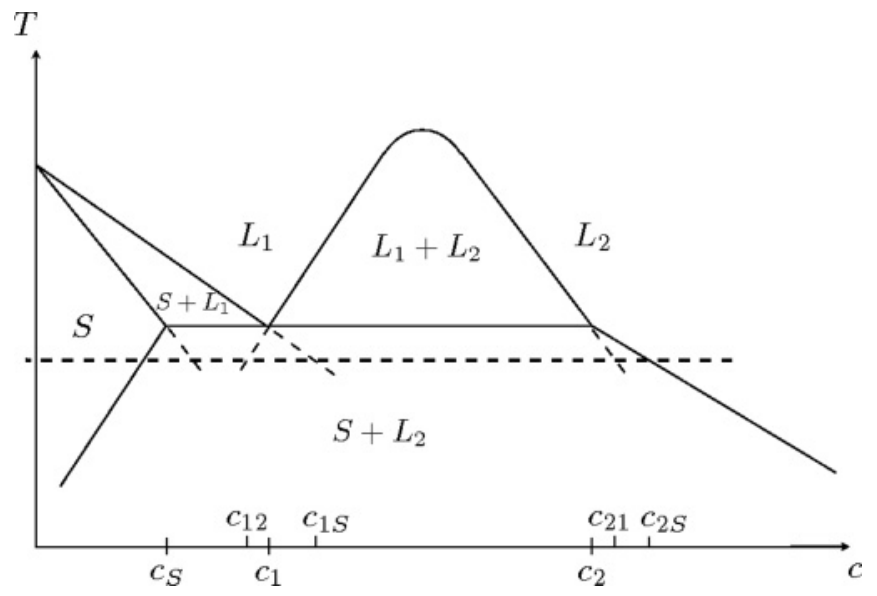

FIG. 1. Phase diagram of the monotectic system.

$L_{1}$ and $L_{2}$ and that the Gibbs-Thomson correction to the equilibrium concentrations $c_{12}$ and $c_{21}$ is the same. These two assumptions allow us to consider the $L_{1}-L_{2}$ interface as a simple source of material in a homogeneous medium that can be treated in the spirit of the symmetric model.

The concentration at any point of the space $(x, y)$ is calculated through an integration over the three curves $y_{S 1}(x)$, $y_{S 2}(x)$, and $x_{12}(y)$, which are given in units of $a$ in the following and represent the three interfaces $S-L_{1}, S-L_{2}$, and $L_{1}-L_{2}$ respectively. Setting the origin of coordinates at the triple junction, the integral reads

$$
\begin{aligned}
I[x, y]= & \frac{P_{a}}{2 \pi} \frac{c_{2}-c_{S}}{c_{2}-c_{1}} \int_{-\infty}^{0} d x^{\prime}\left[2 g\left(x, y ; x^{\prime}, y_{S 2}^{\prime}\right)\right. \\
& \left.-\left(\Delta_{2}-\frac{d_{0}}{a} \kappa\left[y_{S 2}\left(x^{\prime}\right)\right]\right) g^{\prime}\left(x, y ; x^{\prime}, y_{S 2}^{\prime}\right)\right] \\
& +\frac{P_{a}}{2 \pi} \frac{c_{1}-c_{S}}{c_{2}-c_{1}} \int_{0}^{\infty} d x^{\prime}\left[2 g\left(x, y ; x^{\prime}, y_{S 1}^{\prime}\right)\right. \\
& \left.-\left(\Delta_{1}-\frac{d_{0}}{a} \kappa\left[y_{S 1}\left(x^{\prime}\right)\right]\right) g^{\prime}\left(x, y ; x^{\prime}, y_{S 1}^{\prime}\right)\right] \\
& +\frac{P_{a}}{\pi} \int_{0}^{\infty} d y^{\prime} g\left(x, y ; x_{12}^{\prime}, y^{\prime}\right) \frac{d x_{12}^{\prime}}{d y^{\prime}} .
\end{aligned}
$$

The first two terms are the integrals over the solid-liquid interfaces and involve the Green's function

$$
g\left(x, y ; x^{\prime}, y^{\prime}\right)=\exp \left[-P_{a}\left(y-y^{\prime}\right)\right] K_{0}\left(P_{a} \eta\right)
$$

and its derivative

$$
\begin{aligned}
g^{\prime}\left(x, y ; x^{\prime}, y^{\prime}\right)= & \exp \left[-P_{a}\left(y-y^{\prime}\right)\right] \\
& \times\left[K_{0}\left(P_{a} \eta\right)+f\left(x, y ; x^{\prime}, y^{\prime}\right) K_{1}\left(P_{a} \eta\right)\right],
\end{aligned}
$$

where $P_{a}=a v / 2 D$ is a Pećlet number linked to $a, K_{0}$ $\left(K_{1}\right)$ is the modified Bessel function of zeroth (first) order, $\eta=\sqrt{\left(x-x^{\prime}\right)^{2}+\left(y-y^{\prime}\right)^{2}}$, and $f\left(x, y ; x^{\prime}, y^{\prime}\right)=[(x-$ $\left.\left.x^{\prime}\right) d y^{\prime} / d x^{\prime}-\left(y-y^{\prime}\right)\right] / \eta$. The third term on the right-hand side is the integral over the liquid-liquid interface that only involves the Green's function, as explained above. The capillary length $d_{0}$ is assumed, without loss of generality, to be equal for all interfaces. Moreover, we assume that the capillary length is isotropic since, in the presence of

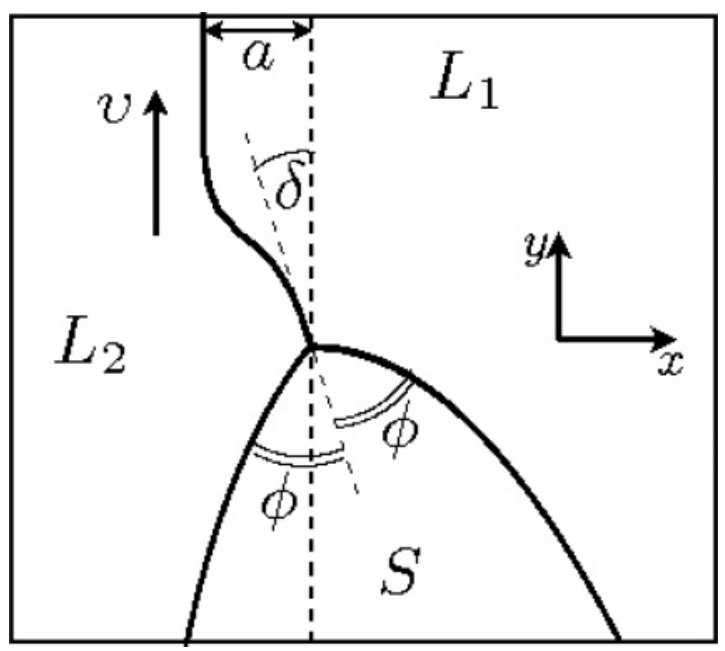

FIG. 2. The solid $S$ grows with velocity $v$ along the metastable liquid-liquid $L_{1}-L_{2}$ interface, which is aligned with $v$ far ahead of the triple junction. In the $x$ direction, the triple junction is shifted by a quantity $a$ from the asymptotic position of the liquid-liquid interface. At the triple junction the $L_{1}-L_{2}$ interface adopts an angle $\delta$ with the velocity direction.

the triple junction, the effect of the anisotropy of surface tension is tiny $[6,7]$. The curvature is conventionally given by $\kappa[y(x)]=-\left(d^{2} y / d x^{2}\right) /\left[1+(d y / d x)^{2}\right]^{3 / 2} . \Delta_{1}=\left(c_{1 S}-\right.$ $\left.c_{12}\right) /\left(c_{1}-c_{S}\right)>0$ and $\Delta_{2}=\left(c_{2 S}-c_{21}\right) /\left(c_{2}-c_{S}\right)>0$ are dimensionless quantities that are related to a Péclet number $P_{i}=v \rho_{i} / 2 D(i=1,2)$ linked to the radius of curvature $\rho_{i}$ of the asymptotically parabolic $S-L_{i}$ front. This relation is due to Ivantsov:

$$
\begin{aligned}
\Delta_{i} & =\sqrt{\pi P_{i}} \exp \left(P_{i}\right) \operatorname{erfc}\left(\sqrt{P_{i}}\right) \\
& \sim \sqrt{\pi P_{i}}, \Delta_{i} \ll 1 .
\end{aligned}
$$

$\Delta_{1}$ and $\Delta_{2}$ are given by the phase diagram (Fig. 1). Close to the monotectic temperature, one can linearize the variations of the equilibrium concentrations and define $\Delta=\Delta_{1} / b_{1}=\Delta_{2} / b_{2}$, where $b_{1}$ and $b_{2}$ are independent of the temperature. $\Delta$ is proportional to the deviation from the monotectic equilibrium temperature.

The solution is found self-consistently by assuming local equilibrium at each interface. In the case of the solid-liquid interfaces $S-L_{i}$ this means

$$
\frac{1}{2} \frac{c_{i}-c_{S}}{c_{2}-c_{1}}\left(\Delta_{i}-\frac{d_{0}}{a} \kappa\left[y_{S i}(x)\right]\right)=I\left[x, y_{S i}(x)\right],
$$

where the factor $1 / 2$ comes from the integration of a Dirac distribution over half of the space [8]. At the liquid-liquid interface, the local equilibrium reads

$$
-\frac{d_{0}}{a} \kappa\left[x_{12}(y)\right]=I\left[x_{12}(y), y\right]
$$

At the triple junction, Young's law imposes two additional constraints, thus allowing the two unknowns $d_{0} / a$ and the dimensionless velocity $v d_{0} / 2 D$ to be determined. The liquid-liquid interface obeys $d x_{12} / d y=-\tan (\delta)$ and the self-selected angle $\delta(>0$ in Fig. 2) is used to 


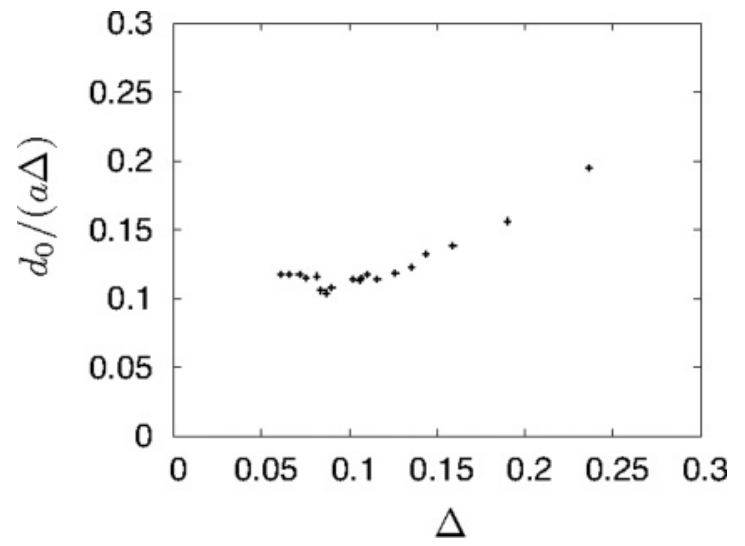

FIG. 3. Plot of $d_{0} / a \Delta$ with respect to $\Delta$.

set the slopes: $d y_{1 S} / d x=1 / \tan (\phi-\delta)$ and $d y_{2 S} / d x=$ $-1 / \tan (\phi+\delta)$, where $\phi>0$ is the contact angle.

One should note that the set of Eqs. (1), (3), and (4) can be derived more formally using the general framework of boundary-integral technique separately in $L_{1}$ and in $L_{2}$ and then combining the two results. Note also that the theoretical framework presented here may be used to describe other types of phase transitions in monotectic systems such as the lamellar growth. After discretization of the interfaces, the resulting set of nonlinear equations is solved by Newton's method complemented by Powell's hybrid method $[9,10]$.

\section{RESULTS}

The solution to the set of equations given above consists of the locus of the interfaces and the values of $d_{0} / a$ and $v d_{0} / 2 D$. In this section we present the results obtained for $\phi=\pi / 3$ and $b_{1}=b_{2}=1$. The latter choice makes our system asymptotically symmetric far behind the triple junction with $\rho_{1}=\rho_{2}=\rho$ since $\Delta_{1}=\Delta_{2}=\Delta$. The miscibility gaps are chosen such that $\left(c_{2}-c_{S}\right) /\left(c_{2}-c_{1}\right)=2$ and $\left(c_{1}-c_{S}\right) /\left(c_{2}-\right.$ $\left.c_{1}\right)=1$.

In Figs. 3 and 4 we present the variations of $d_{0} / a \Delta$ and the dimensionless velocity $v d_{0} / 2 D$ versus $\Delta$, respectively. From these plots one can extract scaling relations in the limit $\Delta \ll 1$ : $d_{0} / a \sim \Delta$ and $v d_{0} / 2 D \sim \Delta^{2}$. This means that $P_{a} \sim \Delta$.

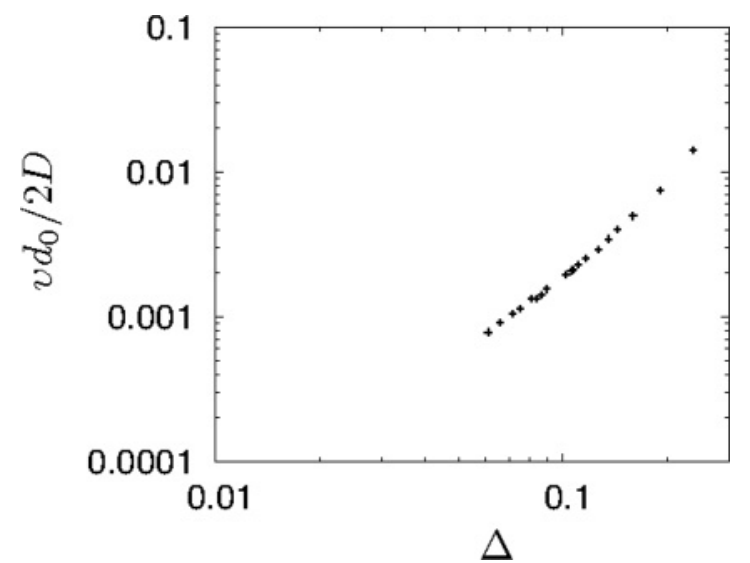
to $\Delta$. (a)
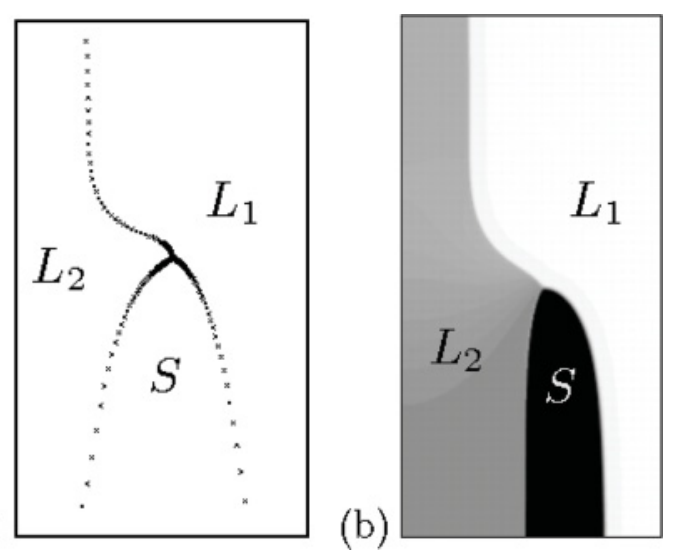

FIG. 5. Steady-state pattern obtained with (a) boundary-integral equations and (b) a phase-field simulation.

According to these scaling relations, the behavior of Eq. (2) for $\Delta \ll 1$ means that $\rho \sim d_{0}$. When $\Delta \rightarrow 0$, the solution in the neighborhood of the triple junction is selected without further matching to the Ivantsov parabola. The curvature of the interfaces is significant only to distances of few $a$ away from the triple junction. The description of the intermediate asymptotics is beyond the scope of this paper. Note that the consistency of the values obtained for $d_{0} / a$ and $v d_{0} / 2 D$ and the pattern in the neighborhood of the triple junction has been tested by changing the size of the system and the boundary conditions for the last points of the calculation.

Finally, we present in Fig. 5(a) the pattern obtained from our calculation for $\Delta=0.215$. In Fig. 5(b) we present also the result of a phase-field simulation. Due to the intrinsic limitation of the parameter space of the phase-field code (which is a modified version of the model developed in Ref. [11] accounting for diffusion in the two liquids), the control parameters are different from the boundary-integral calculation. Our goal here is only to provide support for the stability of the growth along the liquid-liquid interface presented in this paper. Indeed, while the boundary-integral formulation presented here does not discriminate stable and unstable solutions, the phase-field simulation has the advantage of being fully time dependent and prohibits unstable solutions. In contrast, the boundary-integral technique (where only the interface's shape is solved due to the analytical elimination of the diffusion field) requires incomparably less CPU time than the phase-field simulations and facilitates a theoretical analysis.

\section{DISCUSSION}

We discuss now the scaling relations that have been identified in the preceding section for $\Delta \ll 1$ :

$$
d_{0} / a \sim \Delta, \quad v d_{0} / 2 D \sim \Delta^{2} .
$$

First, one can define a quantity $a^{2} v / d_{0} D$ that does not depend on $\Delta$. This invariant has the same form as in classical dendritic growth [12], lamellar eutectic growth [13], or other patternformation processes such as the two-phase finger growth in eutectic systems [14]. Second, according to the behavior of the Bessel functions for small arguments, $K_{0}(z) \sim-\ln z$ and $K_{1}(z) \sim 1 / z$, the scaling relations presented in Eq. (5) mean 
that all terms in Eqs. (1), (3), and (4) are, up to logarithmic corrections, of the same order $\Delta$.

The scaling relations presented in Eq. (5) imply that the two length scales $a$ and $\rho$ are such that $a / \rho \sim 1 / \Delta$. Here one can make an analogy with the physics of the two-phase finger in eutectic systems [14] where a lamella of one solid phase grows together with a second solid phase exhibiting Ivantsov asymptotics. In this eutectic growth (which was observed experimentally in a ternary alloy [15]), apart from the deviation from eutectic temperature, the global concentration of the system is a second degree of freedom. This concentration affects only the radius of curvature of the Ivantsov asymptotics. When the global concentration is of the order of the liquid concentration at the solid-liquid interfaces (eutectic concentration), the width of the lamella is much larger than the Ivantsov radius, similarly to the lateral shift $a$ of the triple junction herein. One might doubt the existence of the two-phase finger close to the eutectic concentration since the lamellar growth is commonly observed in this region of the phase diagram. Here our phase-field simulations tend to prove that the solid finger growing along the liquid-liquid interface is a stable process.

One should note that the process presented in this Rapid Communication is favorable with respect to the growth of a solid dendrite in the bulk of one of the two liquid phases (with a velocity $\Delta^{4}$ ). In a phase-field simulation, a nucleus of solid phase was introduced in the bulk of $L_{1}$ aside from the $L_{1}-L_{2}$ interface. The evolution showed the attraction of the solidliquid interfaces with the liquid-liquid interface and the creation of a triple junction.

Note, finally, that in the patterns shown in Fig. 5, the system is not in full equilibrium far behind the triple junction due to the absence of diffusion in the solid phase. However, a gradient of concentration exists in the solid and a slow drift of the pattern into $L_{1}$ (which disappears at equilibrium) is expected far behind the triple junction when one considers the slow diffusion in the solid.

\section{CONCLUSION}

In this Rapid Communication we study the twodimensional isothermal steady-state growth of the solid phase along the interface between two demixed liquids (liquid-liquid interface) in monotectic systems. Far ahead of the triple junction, the liquid-liquid interface is in a metastable equilibrium and aligned with the velocity direction. In the vicinity of the triple junction, the shape of the interfaces is complex and results from the rejection of impurities from the solid phase into both liquid phases. We develop a boundary-integral formulation, a hybrid between one-sided and symmetric models, which describes diffusion in the two liquids. It is not common practice to use this sort of boundary-integral technique in the context of solidification. We solve the boundary-integral equations for the three interfaces and we present our results depending on the deviation from monotectic temperature $\Delta$. In the limit $\Delta \ll 1$, one can extract scaling relations from our calculations that are supported by analytic arguments. The steady-state velocity scales as $\Delta^{2}$. The length scale $a$ measures, relative to the position of the asymptotic liquid-liquid interface, the shift of the triple junction in the direction perpendicular to the velocity and scales as $1 / \Delta$. We complement our results with a phase-field simulation that proves the stability of the process. The solidification in the monotectic system presented here is an attempt to describe theoretically the growth of a solid finger from a liquid-liquid mixture. We hope our work can stimulate experimental quantitative study of this phase transformation. We also suggest that the theoretical framework presented in this Rapid Communication may be used to describe other types of phase transitions in monotectic systems such as lamellar growth.

\section{ACKNOWLEDGMENTS}

We acknowledge the support of the Deutsche Forschungsgemeinschaft under Project No. SPP1296. We are also grateful to M. Plapp for providing the phase-field code.
[1] L. Ratke and S. Diefenbach, Mater. Sci. Eng. Rep. 15, 263 (1995).

[2] S. R. Coriell, W. F. Mitchell, B. T. Murray, J. B. Andrews, and Y. Arikawa, J. Cryst. Growth 179, 647 (1997).

[3] R. N. Grugel, T. A. Longrasso, and A. Hellawell, Metall. Trans. A 15, 1003 (1984).

[4] B. Nestler, A. A. Wheeler, L. Ratke, and C. Stöcker, Physica D 141, 133 (2000).

[5] G. P. Ivantsov, Dokl. Akad. Nauk SSSR 58, 567 (1947).

[6] D. E. Temkin, Abstracts of ICASP, June 7-10 2005, Stockholm, Sweden (KTH, Stockolm, 2005).

[7] E. A. Brener, C. Hüter, D. Pilipenko, and D. E. Temkin, Phys. Rev. Lett. 99, 105701 (2007).

[8] Y. Saito, G. Goldbeck-Wood, and H. Müller-Krumbhaar, Phys. Rev. A 38, 2148 (1988).
[9] M. J. D. Powell, in Numerical Method for Nonlinear Algebraic Equations, edited by P. Rabinowitz (Gordon and Breach, London, 1970).

[10] J. E. Dennis Jr. and R. B. Shnabel, Numerical Methods for Unconstrained Optimization and Nonlinear Equations (PrenticeHall, New York, 1983).

[11] R. Folch and M. Plapp, Phys. Rev. E 72, 011602 (2005).

[12] J. S. Langer and H. Müller-Krumbhaar, Acta Metall. 26, 1681 (1978); 26, 1689 (1978); 26, 1697 (1978).

[13] K. Kassner and C. Misbah, Phys. Rev. Lett. 66, 445 (1991).

[14] G. Boussinot, C. Hüter, and E. A. Brener, Phys. Rev. E 83, 020601(R) (2011).

[15] S. Akamatsu and G. Faivre, Phys. Rev. E 61, 3757 (2000). 